\title{
Procesos de aprendizaje y terapia de conducta en la adicción a la heroína *
}

\author{
Angel García \\ y José Luis Graña
}

Servicio Piloto de Drogodependencias Vallecas-Mediodia

Ayuntamiento de Madrid

\section{INTRODUCCION}

El sector de población más afectado por la heroína oscila entre los 15 y 35 años. El consumo se inicia por vía inhalante (esnifado por la nariz) y al cabo de dos o tres años se adopta la vía intravenosa. El adicto suele ser un politoxicómano, combina cocaína con heroína, ingiere grandes dosis de alcohol mientras prosiguen los efectos de la heroína, etc. La vía de administración intravenosa facilita la aparición de procesos infecciosos como hepatitis, bronquitis, etc; alteraciones inmunológicas graves como el Síndrome de Inmunodeficiencia Adquirida (SIDA) y el riesgo de sobredosis mortal aparece a veces por un fallo en la tolerancia del organismo.

Debido a la tolerancia, las necesidades diarias de heroína se incrementan progresivamente y en pocos años se puede alcanzar un consumo de uno a dos gramos en cuatro a seis dosis. La dependencia tiene un componente fisiológico y otro psicológico, llamado querencia o deseo (CRAVING). El síndrome de abstinencia se manifiesta con unos síntomas de rebote o contrarios a los efectos de los opioides: ansiedad, lagrimeo, escalofríos, bostezos, diarreas, insomnio, etc. (Jaffe, 1982).

Las primeras experiencias con la heroína suelen ser poco agradables, se producen náuseas, vértigos, un despertar similar a la resaca del alcohol; pero a las cuatroseis experiencias los efectos eufóricos, placenteros y analgésicos se hacen predominantes; estableciéndose rápidamente la dependencia física y psíquica.

La farmacología molecular ha permitido descubrir en el cerebro, médula adrenal, etc., receptores específicos para los opiáceos. La localización de los receptores se corresponde con dos de los principales efectos de la heroína, la euforia y la analgesia. Las moléculas con carácter morfínico endógeno se denominan encefalinas y endorfinas. El papel de la heroína, una vez que se transforma en morfina, sería la de actuar en los receptores de los opiáceos endógenos y sustituir el papel fisiológico de los opiáceos endógenos (endorfinas y encefalinas) (Snyder, 1984).

Cuando se ha impuesto el «estilo de vidan de heroinómano, la actividad de este

\footnotetext{
*Agradecimientos: Julián Alvarez, Remedios Comas, Almudena Gozalo, Jesús Ortiz, Mercedes Pereira.
} 
gira alrededor de la heroína; por tal motivo, el toxicómano trafica, roba, se prostituye, etc.

La perspectiva médica ha visto al adicto a la heroína como un enfermo metabólico a una sustancia química generando programas de mantenimiento de metadona como sustitutivo de la heroína (Dole, 1981). A nivel farmacológico, en los países anglosajones, también se han introducido el uso de antagonistas (ciclazocina, naltrexona), con este tipo de tratamiento, teorícamente, al administrarse heroína y estar bloqueados los efectos de ésta, termina por desaparecer la adicción.

Otra alternativa la han constituido las Comunidades Terapéuticas de exadictos como El Patriarca, Narconón, etc. El problema central es el regreso del adicto a su medio social y evitar el proceso de recaída.

La perspectiva conductual ha analizado profundamente aspectos como la implicación de los principios de aprendizaje en la adicción a la heroína, la evaluación conductual, el estudio de procesos específicos, la terapia de conducta y los aspectos metodológicos a adoptar para realizar mejores investigaciones.

\section{PRINCIPIOS DE APRENDIZAJE Y ADICCION A LA HEROINA}

Los efectos de la heroina como reforzador positivo tienen características hedónicas: generan sensaciones intensas de euforia, placer, bienestar y ciertas connotaciones más potentes y duraderas que el orgasmo, según los propios heroinómanos. Los primeros ensayos de autoadministración de heroína son mantenidos por este reforzamiento positivo, pero las interacciones de condicionamiento clásico y operante son más potentes y complejas que en problemas conductuales como una fobia, depresión, etc.

La inyección de heroína constituye un EI farmacológico que elicita una serie de respuestas psicológicas y farmacológicas (euforia, analgesia, etc.) RI. Los estímulos ambientales presentes en los ensayos de autoadministración se conforman en $E C$ Compuestos (jeringuilla, ritual, servicio en el que se pincha), tales estímulos poseen una saliencia o intensidad diferente y adquieren una doble capacidad para elicitar $R C$ : por una parte respuestas funcionalmente parecidas a las de la heroína, $R C S_{i-}$ milares (ejemplo: la "fantasía de la aguja» consiste en sentir la aguja en la vena, bombeando sangre con agua e inyectarse. Con ello se alcanza un clímax similar al flash de la heroína) y por otra la de evocar $R C$ Compensatorias de signo opuesto a los efectos de la heroína, dándose en este segundo caso un síndrome de abstinencia condicionado (Siegel y MacRae, 1984; Grabowski y O'Brien, 1981) en estrecha correlación con $E C$ ambientales. Clínicamente se reflejan estas $R C$ Compensatorias al exponerse al heroinómano a condicionamiento encubierto, prevención de respuesta con el «ritual», etc. Las manifestaciones subjetivas y fisiológicas que se dan con deseo o querencia de heroína, ansiedad, náuseas, escalofríos, bostezos y una actuación de los indicadores psicofisiológicos de frecuencia cardíaca, frecuencia respiratoria, tensión sanguínea, etc.

Wikler (1980), además de señalar la importancia del refuerzo farmacológico y psicológico positivo de la morfina, resalta el papel de los $E C$, el experimentar en la calle repetidos síndromes de abstinencia se constituirá en un reforzador negativo, haciendo que el heroinómano busque el alivio de la ansiedad que produce tal estado. Realiza el primer análisis de las recaídas después del tratamiento en base a los $E C$ ambientales, deseo o querencia de heroína y la conducta de búsqueda. Para el proceso terapéutico, el eje central está en la resistencia a la extinción, siendo preciso extinguir los $E C$ y el síndrome de abstinencia condicionado, con técnicas de terapia de conducta y el uso de antagonistas farmacológicos. Combinando la terapia de conducta con fármacos bloqueadores de la heroína, al pincharse el condicionamiento clásico de autoadministración de heroína.

Algunos de los principios de condicionamiento operante más importantes son:

$E l$ "ritual, constituye un encadenamiento que finaliza en el refuerzo positivo que produce la heroína. De un heroi- 
nómano a otro se observan ciertas diferencias topográficas en esta cadena de conducta.

El "argot» (caballo, mono, camello), constituye un reforzador condicionado más.

El "estilo de vida», propio del heroinómano, con la secuencia de levantarse tarde por la mañana, salir de casa y contactar con el camello, el ritual de preparación y el efecto de la heroína constituye un programa de reforzamiento de segundo orden (Goldberg, Kelleher, Morse, 1975).

En este tipo de programas breves, estímulos ambientales están presentes en largas secuencias conductuales de búsqueda y toma de drogas, mantenidas por el reforzador terminal de la heroína.

El uso de la heroína por el adicto está sometido a programas de reforzamiento altamente intermitentes (la calidad de la heroína varía de unas veces a otras y su efecto temporal oscila entre dos-cinco horas). Unas veces se administra iniciados los primeros síntomas de abstinencia y otras veces no. Todo esto hace que interaccionen distintos programas de reforzamiento intermitente que inciden, sobre el efecto de la droga, la poca tolerancia a la frustración y en el estilo de vida del adicto (Kaplan, 1983).

En general, podemos resumir las contingencias de reforzamiento, del siguiente modo:

a) Reforzamiento positivo asociado con aspectos sociales del uso de la heroína (ejemplo: aceptación en el grupo de heroinómanos, una cultura del uso de la heroína, estilo de vida, etc.).

b) Reforzamiento positivo asociado con las propiedades farmacológicas de la droga (euforia, bienestar, el experimentar el flash inicial, etc.).

c) Reforzamiento negativo asociado con aspectos aversivos del ambiente (aliviar la monotonía, reducir aspectos aversivos de la vida familiar y demás condiciones de la vida laboral, personal, etc).

d) Reforzamiento negativo vinculado a estados físicos aversivos inducidos por la heroina (ejemplo: alivio del malestar físico del síndrome de abstinencia).

\section{EVALUACION CONDUCTUAL}

Los procesos de evaluación, de abuso de la heroína, cubren un historial médico, autorregistros conductuales, estudios de laboratorio, informes de asistentes sociales, judiciales y policiales.

La evolución conductual, propiamente psicológica, es un proceso continuo y el abuso de las drogas se evalúa antes, durante y después del tratamiento. Los métodos de medición se realizan a nivel conductual, cognitivo y fisiológico (Van Hasselt, Milliones, Hersen, 1981). Estos autores resaltan la necesidad de realizar autorregistros y una evidencia corroborativa de estos análisis de orina que les dan validez externa, registros psicofisiológicos ante estímulos condicionados vinculados al abuso de la heroína, estimaciones bioquímicas cuantitativas de opiáceos y otras drogas, etcétera.

a) Autorregistros. Los procedimientos de autorregistro se aplican en programas de tratamiento de amplio espectro (Boudin, et al., 1977), midiéndose la frecuencia de "urgencias" de consumo de heroina, pensamientos de frustración, etc. Se evalúan, también, indicadores laborales, de escolaridad, etc., de un modo diario. Los clientes son requeridos a contactar con miembros del equipo (staff) diariamente, llamando por teléfono cada tres horas. $\mathrm{La}$ validez externa del autorregistro se obtiene de fuentes como oficinas de empleo, juzgados, asistentes sociales, etc.

El estudio de validez del autorregistro del heroinómano, con criterios externos como el test cualitativo de orina (consuma o no opiáceos), informes de asistentes sociales, policiales, etc., revelan sorprendentemente una concordancia superior al 70 por 100 (Amsel, et al., 1976; Ball, 1967).

b) Estudios de autoadministración en situaciones de laboratorio. Adictos de «calle» que son admitidos en un Centro Experimental han sido estudiados en distintos tipos de condiciones, que incluyen:

1. Períodos de abstinencia de droga.

2. Autoadministración de heroína 
bajo condiciones experimentales ciegas, con y sin uso de antagonistas o bloqueadores de heroína.

3. Administración de metadona.

4. Administración de naltrexona.

Estas investigaciones se realizan en situaciones altamente controladas y con uso de estadísticas multivariadas (Meyer y Mirin, 1979).

La conducta operante consistía en trabajar con una palanca de mano variando el coste de heroína según el experimento. Los resultados indican que la autoadministración de heroína (refuerzo positivo) es facilitado por la conducta operante. El uso de antagonistas (naltrexona) reducía considerablemente la autoadministración de heroína después de realizar la tarea operante.

c) Estudios psicofisiológicos. Analizan el papel de los estímulos condicionados vinculados a la administración de opiáceos, se registran respuestas como diámetro de la pupila, $R G P$, frecuencia cardía$\mathrm{ca}$, frecuencia respiratoria, temperatura y deseo o querencia de droga (CRAVING).

Wikler (1971), señaló el principio teórico para la realización de estos estudios, al informar que el emparejamiento de síntomas de abstinencia con estímulos ambientales resultan en la elicitación de un síndrome de abstinencia condicionado.

$\mathrm{El}$ estudio del síndrome de abstinencia condicionado se desarrolló en situaciones altamente controladas (O'Brien, 1977), siendo publicados los resultados en la prestigiosa revista Science. Un grupo de heroinómanos que estaban en mantenimiento con metadona, recibieron voluntariamente una inyección de naloxona (un antagonista que precipita los síntomas de abstinencia). Antes de la aparición de los síntomas, los pacientes oían un estímulo auditivo combinado con un olor a menta. Después de 6 a 11 ensayos, los síntomas de abstinencia aparecían al exponerse los sujetos al sonido y olor combinados, independientemente de que se les hubiera administrado o no naloxona. La abstinencia subjetiva (quejas de náuseas y escalofríos) se dio en todos los sujetos. En la inmensa mayoria se dieron además cambios fisiológicos como llantos, jadeos, incremento en la frecuencia respiratoria y cardíaca, incremento de la tensión y dilatación de las pupilas y descenso de la temperatura de la piel. De este modo, un estímulo neutral puede condicionarse para que elicite síntomas de abstinencia (RC COMPENSATORIAS) y también deseo o querencia de droga (CRAVING).

d) Signos físicos. Otro procedimiento de evaluación lo constituyen los cuestionarios físicos del síndrome de abstinencia que se miden durante la desintoxicación física y en el tratamiento psicológico, valorizándose únicamente la presencia o ausencia de estados de ansiedad, deseo o querencia de droga, bostezos, lagrimeo, insomnio, temblores en piernas y/o brazos, náuseas, vómitos, etc.

Las variables fisiológicas que se miden en estos cuestionarios son: tensión arterial, pulso, temperatura y frecuencia respiratorias.

Este tipo de registros los suelen realizar ATS o auxiliares de clínicas, pero es un procedimiento de valoración más a tener en cuenta en la intervención psicológica.

e) Análisis bioquímicos. En los programas de intervención con heroinómanos, se realizan estudios periódicos cuantitativos de consumo de opiáceos, cocaína, anfetaminas, etc., mediante procedimientos de cromatografía o de banda fina. También se valoran análisis cualitativos de orina (positivos o negativos), que sirven para valorar la adherencia al tratamiento; los análisis cualitativos se suelen realizar al azar, para que el paciente no tenga ninguna clave temporal.

f) Patronatos de uso y abuso de drogas. El «fenómeno de escalada» ha sido analizado mediante estudios longitudinales con uso de la técnica de panel y el escalograma de Guttman, examinándose los factores determinantes del paso de la fase «ocasional» o «experimental» de una droga a un uso intensivo y regular (Kandel, 1978). Los factores más importantes son:

1. Criterio de edad. Cuanto más temprano es el contacto con la droga, mayor es la posibilidad de pasar a una condición de toxicómano regular. A partir de los 
veinticinco años el riesgo ha concluido.

2. Comportamiento y sistema de valores. El fracaso escolar, delincuencia juvenil, bajo status social y el abuso de drogas legales (café, tabaco, alcohol) precede generalmente al uso de drogas ilegales.

3. Características psicológicas. Los síntomas de depresión presentan una correlación muy importante con el paso de la droga blanda a una droga dura, así como el predominio de actitudes sociales de rebeldía y rechazo.

4. Actitudes y comportamientos de los padres. La tolerancia de los padres y sus creencias en el carácter inofensivo de las drogas, facilitan el desarrollo de la toxicomanía en los hijos. El ejemplo de los padres bebedores de alcoholes fuertes en graduación, permite preveer el mismo proceso de aprendizaje social en sus hijos. Por último, una falta de comunicación verbal en la familia, entre padres e hijos, es otro factor de riesgo.

El análisis de estos componentes confir- ma el carácter multifactorial del desarrollo de la condición de toxicómano. De hecho la investigación psicosocial se ha localizado en variables sociales como uno de los factores más importantes, resultando la conducta de "modelling" familiar y de grupo como una variable crítica para la entrada en contacto con los opiáceos.

En el patrón de toma de opiáceos se han encontrado variaciones muy interesantes en los patrones de uso (Zinberg et al., 1978): adictos diarios de los opiáceos, adictos físicamente dependientes pero con un funcionamiento social adecuado, politoxicómanos en los que la heroína es una más de las drogas a consumir y consumidores controlados (de fines de semana), que desarrollan ciertas reglas para evitar el desarrollo de la dependencia.

\section{TERAPIA DE CONDUCTA CON HEROINOMANOS}

El tratamiento conductual de heroinómanos se inicia después de la desintoxicación física, adaptándose distintas estrategias de condicionamiento operante (con- tacto de contingencias y economias de fichas) y programas multimodales.

\section{Condicionamiento clásico}

El terapeuta considera como $E C$ los lugares donde se pincha, el ritual de preparación y material que se usa (jeringuilla, papelina, cucharilla). Todos estos estímulos, elicitan dos tipos de $R C$ (similares y compensatorias). Las RC COMPENSATORIAS se presentan como sindrome de abstinencia condicionado ante estímulos audiovisuales (diapositivas y vídeos) relacionados con el ritual (Teasdale, 1973; Grabowski, O’Brien, et al., 1980). Dos tipos de tratamiento se han aplicado para reducir o eliminar el uso de la heroína:

a) Contracondicionamiento. Aversión química y eléctrica. Condicionamiento encubierto. Desensibilización Sistemática in vivo.

Mediante el condicionamiento aversivo se tratan de modificar las respuestas que están vinculadas a EC de la heroína. En su aplicación, un estímulo aversivo (químico, como la naloxona, o eléctrico, como un shock débil) se empareja con la conducta de inyectarse y el refuerzo de la heroína, con pensamientos e imágenes del ritual y de los efectos de la heroína y con la parafernalia usada en la preparación y en el ritual de inyectarse. Mediante este tipo de terapia, los $R C$ originales evocadas por conductas, pensamientos y estímulos vinculados a la heroína pueden romperse y elicitarse respuestas aversivas condicionadas como ansiedad, náuseas, malestar físico, etc.

Por facilidad de aplicación se ha impuesto el procedimiento de condicionamiento encubierto, usándose para producir una respuesta de evitación condicionada a conductas vinculadas con el deseo, búsqueda y uso de heroína (Steinfeld, 1970). El paciente puede ser entrenado a usar esta técnica en situaciones naturales, aunque la validez de los autoinformes de este tipo de técnica se cuestiona.

La desensibilización sistemática se ha usado con politoxicómanos, en los que la 
ansiedad social es el factor central en el consumo de drogas (Kraft, 1968), realizándose desensibilización in vivo con las escenas que evocan ansiedad en situaciones sociales.

Se han estandarizado jerarquías de situaciones estimulares que provocan deseo, búsqueda y uso de heroína (O'Brian et al., 1983). Con ello se han puesto las bases para establecer una desensibilización progresiva imaginada o in vivo. El problema central sigue siendo el grado de generalización al medio natural del toxicómano.

b) Extinción. Antagonistas y extinción.

Wikler (1983) sugirió la necesidad de extinguir el síndrome de abstinencia condicionado, así como la conducta de deseo, búsqueda y uso de heroína.

El uso de antagonistas de los narcóticos, como la ciclazocina y la naltrexona, se han combinado con el procedimiento de extinción. Mientras el adicto está en tratamiento con antagonistas, puede exponerse a estímulos ambientales que provocan el uso de opiáceos y hasta el uso de heroína, sin experimentar el reforzamiento farmacológico de éste. Pero el paciente no realiza el ritual que precede al inyectarse heroína, de ahí que no se hayan extinguido las respuestas condicionadas asociadas con esta conducta. Por optimizar el uso del tratamiento con antagonistas se introducen contingencias económicas por su uso (Grabowski et al., 1979).

\section{Condicionamiento operante}

Se ha aplicado en tratamientos cerrados $y$ en situaciones externas con heroinómamos. Las técnicas más usadas son el contrato de contingencias entre el paciente $y$ el terapeuta, especificándose qué conductas hay que incrementar o reducir en frecuencia y las consecuencias reforzantes o aversivas. En los tratamientos cerrados, se introducen sistemas de economía de fichas para obtener privilegios o refuerzos.

\section{a) Contrato de contigencia}

En este tipo de estudios el objetivo central es el descenso de frecuencia en el uso de heroína; otras conductas, como mejorar el cumplimiento de las normas que rigen el centro, la asistencia a sesiones de terapia de grupo, el realizar actividades físicas y reducir los pensamientos subjetivos de frustración, se introducen en los contratos. Sin embargo, se ha encontrado que los reforzadores introducidos en este tipo de programas son menos potentes que los inherentes a la toma de heroína (Beatty, 1975).

El contrato de contingencias suele incorporarse a los tratamientos de mantenimiento con metadona. El tipo de reforzador que se usa es la toma de metadona en casa determinados días, al ir más periódicamente a la clínica. De hecho se destinan a lograr una mejor adherencia conductual al mantenimiento con metadona (Stitzer y Bigelow, 1978).

\section{b) Economia de fichas}

Este tipo de programas se dirigen más. a las conductas del paciente dentro de la situación residencial que al problema de la abstinencia en el consumo de drogas. Así se ha aplicado el principio de Premack con pacientes heroinómanos, haciendo que actividades de alta probabilidad de ocurrencia como recibir visitas, ver la TV, etc., sean contingentes con conductas de baja probabilidad como puntualidad, cumplir las normas de la residencia, etc. (O'Brian, Raynes y Patch, 1971).

\section{Tratamientos multimodales}

Los programas de amplio espectro se revelan como los más importantes en las aplicaciones terapéuticas. Así, se ha sugerido (Callner y Ross, 1980) que el mantenimiento de una condición libre de drogas depende de la extinción del deseo de droga, del desarrollo de múltiples habilidades sociales y de reinserción en un medio libre de drogas y reiniciar un nuevo estilo de vida.

Los objetivos se han dirigido hacia extinguir la conducta de deseo o querencia 
de heroína mediante uso de relajación progresiva, sensibilización encubierta y manejo de la ansiedad. Entrenamiento en habilidades sociales para rehusar las ofertas de heroína en el medio natural, habilidades sociales para encontrar empleo y solución de problemas. Manejo de contingencias para establecer la abstinencia en el uso de heroína medio mediante análisis de orina.

Se usan diferentes técnicas conductuales para facilitar el autocontrol en pacientes mantenidos con metadona, tales como relajación, desensibilización, ensayo conductual, entrenamiento de autoimagen. Se evalúa a los pacientes en ansiedad, locus de control, autoimagen y asertividad con la finalidad de evaluar los efectos de los programas. Los análisis estadísticos prepostratamiento revelan un descenso en los niveles de ansiedad, y un incremento en el nivel de autoestima y facilitación del control interno (Cheek et al., 1973).

También se ha investigado el papel que juegan los déficit en habilidades sociales en heroinómanos. Muchos de ellos usan la droga para evitar la ansiedad generada por un inadecuado funcionamiento social, de ahí que el entrenamiento en habilidades sociales tenga dos componentes básicos: un entrenamiento en asertividad y entrenamiento conductual para acceder al mercado de trabajo (Van Hasselt, Hersen y Milliones, 1978).

\section{METODOLOGIA}

Para una evaluación de resultados es prioritario dividir en áreas la intervención conductual. Se han sugerido cuatro tipos de tratamientos (Stitzer y MCaul, 1983):

a) Tratamientos localizados en el control de estímulos del uso de drogas. Este tipo de intervención conductual tiene lugar en centros cerrados para toxicómanos, con ellos se modifican la disponibilidad en el medio social del heroinómano y se introducen programas de condicionamiento operante.

b) Tratamientos localizados en alterar la potencia reforzante de la droga. Este tipo de tratamiento se concreta en programar el mantenimiento con metadona $y$ contrato de contingencias, o bien el uso de antagonistas como la naltrexona y la extinción de la dependencia psicológica.

c) Tratamientos que introducen únicamente contingencias conductuales. Se introducen programas de intervención para la deshabituación psicológica, manejo de ansiedad, terapia de pareja y/o familiar, etcétera.

d) Tratamientos localizados en habilidades sociales y en promover una reinserción social, con técnicas conductuales de entrenamiento en habilidades sociales, solución de problemas acceso al mercado de trabajo.

A nivel de medición es preciso desarrollar dos tipos de técnicas: Una medición conductual del programa por componentes, con el fin de evaluar la ejecución en distintas tareas mientras el paciente está en tratamiento, estudiando el manejo de situaciones y dificultades en las que se encontrará éste al finalizar dicho tratamiento. Un ejemplo es el prearreglo de una situación como la oferta de heroína. Esto puede ser más útil para la terapia que el autoinforme del paciente.

El otro tipo de medición consiste en la evaluación in vivo de conductas en situaciones naturales. Aquí es fundamental aislar medidas vinculadas a la droga y conductas postratamiento. Ejemplo de estas medidas serian: los informes de compañeros no vinculados a la droga, vigilar y evitar situaciones de alto riesgo, como pasarse por las zonas donde antes obtenía la heroína, etc. De hecho, para la transferencia a situaciones naturales es precisa la formación de grupos de autoayuda de ex toxicómanos (del tipo de alcohólicos anónimos) y un entrenamiento cognitivo-conductual en prevención de recaídas (Marlatt, 1979).

La dependencia de la heroína introduce múltiples problemas sanitarios, legales, familiares y psicológicos, de ahí que sea precisa una intervención comunitaria, psicológica y médica. Es necesario un seguimiento continuo después de la intervención, pues el 90 por 100 de las rècaídas acontecen en los seis primeros meses después del tratamiento. 
El introducir la medición multivariada permite analizar el porcentaje de variación total con que contribuyen los programas de deshabituación psicológica, entrenamiento en comportamiento social, preven- ción de recaídas, etc., así como aislar los componentes experimentales de cada programa mediante una metodología de diseño experimental de grupos.

\section{Resumen}

Múltiples principios de condicionamiento clásico y operante interaccionan en la adición a la beroina. Destacan la presencia de RC Compensatorias en forma de Sindrome de Abstinencia Condicionado (SAC) y distintos programas de reforzamiento en el estilo de vida, argot y efectos de la heroína. En evaluación conductual se estudian los correlatos subjetivos y psicofisiológicos del SAC; la validez externa de los autorregistros se logra mediante análisis de orina. En terapia de conducta los programas de amplio espectro se combinan con la intervención comunitaria y apoyo médico. A nivel metodológico se resalta la necesidad de usar múltiples criterios de medición, introducción del análisis multivariado y uso de diseños estadísticos, realización de seguimientos a largo plazo. Palabras claves: Heroína, Terapia de Conducta, Evaluación Conductual, Condicionamiento Clásico y Operante, Síndrome de Abstinencia Condicionado.

\section{Summary}

Learning processes and behavior therapy in beroin addiction.

Multiple principles of classical conditioning and operant behavior interact in the addiction to beroin.

Among these, the presence of conditioned compensatory response stands out, in the form of conditioned abstinence syndrome. Different schedules of reinforcement determine life style, Lang, effect of heroin, etc.

At the level of behavior assessment most emphasis has been placed on the subjective and psychophisiological respon:os associated with conditioned abstinence syndrome.

The external validity of seif-monitoring is achieved by urine analysis; at the level of behavior therapy wide spectrum programmes have been developed which combine comunity intervention with medical support.

Metbodologically, the need to use multiple criteria of measurement is important, as is the introduction of multivariuded analisis, the used of statistical design, and finally, the carryng-out of long term follow-up studies.

KEY WORDS: Heroin, Behavior Assesment, Bebavior Therapy Conditioning, Conditioned Abstinence Syndrome.

\section{Referencias}

Amsel, Z.; Mandell, W.; Matthias, L.; Mason, C., y Hocherman, I.: «Reliability and validity of selfreported illegal activities and drug use collected from narcotic addicts». Inter. J. Addict, 11, 325-336, 1976.

Ball, J. C.: *The reliability and validity of interview data obtained from 59 narcotic drug addicts». Am. J. Sociol., 72, 650-654, 1967.

BEATTY, J.: The effect of contingency behavioral contracting with beroin addicts. Ph D. Dissertation, Rutgers University, 1975.

Boudin, H.; Valentine, V.; Ingram, R.; Brantley, J.; Ruiz, M.; Smith, G.; Catlin, R., y Regan, E.: "Contingency contracting with drug abusers in the natural environment". Inter. J. Addict, 12, 1-16, 1977.

CALLNER, D., y Ross, S.: *The assessment and training of assertion skills with drug addicts: A preliminary study». Inter. J. Addict, 13, 227-239, 1978. 
CAllneR, D., y Ross, S.: «Behavioral treatment approach to drug abuse». En J. M. Ferguson y C. B. Taylor: The comprebensive handbook of behavioral medicine, vol. 3, New York, MTP, Press Limited, 1973.

Cheer, F.; Tomarchio, T.; STANDEN, J., y Albahary, R.: *A behavior modification training program en self-control for addicts on methadone maintenance». Intern. J. Addict, 8, 969-996, 1973.

DOLE, V.: «La conducta adictiva». Investigación y Ciencia, 53, 68-75, 1981.

Goldberg, S.; Kelleher, R., y MORSE, W.: "Second-order schedules of drug injection». Fed. Proceed., 34, 1771-1772, 1975.

Grabowski, J., y O'BRIEN, Ch.: «Conditioning factors in opiate use». En N. K. Mello (Ed.): Advances in substance abuse. Behavioral and Biological Research, vol. 2, Greennwich, Connecticut, Jai Press, 1981.

JAFFE, J.: «Drogadicción y abuso de drogas». En A. Goodman, L. S. Goodman y A. Gilman (Ed.): Las bases farmacológicas de la terapéutica. Buenos Aires, Médica Panamericana, 1982.

KANDEL, D. B.: Longitudinal research on drug abuse. New York, John Wiley, 1978.

Kaplan, J.: The hardest drug: Heroin and public policy. Chicago, University Chicago Press, 1983.

Kraft, T.: «ocial anxiety and drug addiction». Br. J. Soc. Psychiatry, 2, 192-195, 1968.

MARLATT, G.: "A cognitive-behavioral model of relapse process". En N. A. Krasnegor (Ed.): Bebavioral analysis and treatment of substance abuse. NIDA. Research Monograph n. 25 . Washington D.C.: U.S. Government Printing Office, 1979.

MEYER, R., y MIRIN, S.: The beroin stimulus. Implications for a theory of addiction. New York, Plenum Press, 1979.

O'Brien, Ch.; Testa, T.; O’Brien, T.; Brady, J., y Wells, B.: Conditioned narcotic Withdrawal in humans. Science, 195, 1000-1002, 1977.

O'Brien, Ch.; Greenstein, R.; TeRnes, J.; MCLellan, A., y Grabowskı, J.: “Unreinforced self-injection: Effects on rituals and outcome in heroin addicts". En L. S. Harris (Ed.): Problems of drug dependence, 1979. NIDA Research Monograph n. 27. Washington D.C.: U.S. Gobernment Printing Office, 1980.

O'Brien, Ch.; Ternes, J.; Greenstein, R.; GrabowsKı, J., y WoOdy, G.: "Aplicaciones de la farmacología conductual humana a los problemas de los adictos a las drogas: Una breve revisión». En V. M. Alcaraz, V. A. Colotla y U. G. Laties (Ed.): Drogas y Conducta, México/Trillas, 1983.

O'BRIEN, Ch.; RAYNES, A. E., y PATCH, V. D.: "Ân operant reinforcement system to improve ward behavior in in-patient drug addicts». J. Behav. Ther. Exper. Psychiatry, 2, 239-242, 1971.

SIEGEL, S.; MCRAE, J.: «Environmental specifity of tolerance». Trend in Neurosciences, 140-143, May, 1984.

SNYDER, S. H.: "Drugs and neurotransmitter receptors in the brain". Science, 224, 22-31, 1984.

STEINFELD, G.: "The use of covert sensitization with institucionalized narcotic addicts". Intern. J. Addict, $5,225-232,1970$.

STITZER, M., y BIGELOw, G.: «Contingency management in a methadone maintenance programs: Availability of reinforcers». Inter. J. Addict, 3, 737-746, 1978.

STITZER, M.; BigelOw, G., y MCCAUL, M.: «Behavioral approaches to drug abuse». En M. Hersen, R. M. Eisler y P. M. Miller (Ed.): Progress in behavior modification, vol. 14. New York, Academic Press, 1983.

TEASDALE, J.: "Conditioned abstinence in narcotic addicts". Intern. J. Addict, 8, 273-292, 1973.

VAN HASSElt, V.; HeRsen, M., y Milliones, J.: «Social skills training for alcoholics and drug addicts: A review». Addictive Behavior, 3, 221-223, 1978.

VAn Hasselt, V.; Milliones, J., y Hersen, M.: «Behavioral assessment of frug addiction: strategies and issues in research and treatment". Intern. J. Addict., 16, 43-68, 1981.

WIKLER, A.: "Present status of the concept of drug dependence". Psychol. Med., 1, 377-380, 1971.

WIKLER, A.: «Dynamics of drug dependence: Implications of a conditioning theory for research and treatment». Archives of General Psychiatry, 28, 611-616, 1973.

WIKLER, A.: Opioid dependence: Mechanisme and treatment. New York, Plenum Press, 1980.

Zinberg, N.; Harding, W.; Stelmack, S., y Marblestone, R.: Patterns of heroin use. Annals of the New York Academy of Sciences, 311, 10-24, 1978. 